laïque à la veille de la Révolution tranquille. De plus, l'auteure et les auteurs du livre mettent à la disposition de la lectrice et du lecteur des photos, des annexes remplies de données historiques ainsi qu'une chronologie des évènements qui complètent la riche documentation de l'ouvrage. Cette publication intéressera tout autant le grand public que les chercheuses et les chercheurs, les étudiantes et les étudiants en formation à l'enseignement et les enseignantes et les enseignants en exercice voulant en connaitre davantage sur l'histoire singulière de cette profession au Québec.

Adriana Morales-Perlaza

Université de Montréal

\title{
Susanne Commend
}

Vulnérables, tolérés, exclus: Histoire des enfants handicapés au Québec, 1920 1990

Montréal : Presses de I’Université de Montréal, 2021. 256 pp.

L'histoire de l'enfance demeure sous-explorée; cela tient en partie aux difficultés inhérentes à la recherche documentaire et au peu de sources générées par des enfants préservées dans les archives. Il s'agit néanmoins d'une période de la vie sur laquelle les sociétés projettent systématiquement leur vision d'elles-mêmes et de leur avenir, ce qui en fait un objet d'étude utile pour faire ressortir les valeurs sociales dominantes pendant la période. Ce qui est vrai de l'enfance en général l'est davantage encore lorsqu'il s'agit de l'enfance "exceptionnelle ", dont l'étude permet d'affiner le regard pour considérer les valeurs sociales projetées sur une catégorie de personnes alternativement considérées "vulnérables, tolérées, ou exclues ». Susanne Commend explore l'univers des enfants handicapés au Québec de la fin de la Première Guerre mondiale aux années 1990 en portant un regard particulier sur l'image complexe et changeante de l'enfant handicapé en lien avec les transformations sociales au Québec dans la même période. La valeur de cette étude dépasse donc le seul champ de l'histoire du handicap.

L'étude de Commend s'étend sur cinq chapitres à la fois thématiques et chronologiques qui permettent d'explorer le sujet dans son évolution et dans ses continuités. Il en ressort trois « logiques » tirées d'un modèle de Marie-Claire Cagnolo, auxquelles correspondent trois époques marquées par une certaine cohérence dans les attitudes sociales face au handicap. La première de ces logiques, qui illustre le qualificatif « vulnérables » du titre, est marquée par une représentation de l'enfant handicapé comme objet de charité et où les intervenants principaux relèvent de la philanthropie, en collaboration avec l'État. Durant cette période, qui s'étend de 1920 à 1960, la principale source de handicap physique chez les enfants est la poliomyélite. La Première Guerre mondiale ayant contribué à une valorisation de l'enfance et à de nouvelles politiques d'assistance publique, les enfants subissant des handicaps physiques des suites de la polio deviennent l'objet d'une attention nouvelle, car ils sont investis 
d'une importance symbolique sans précédent. En ce sens, les philanthropes profitent de cette représentation de l'enfance innocente et vulnérable pour financer et soutenir hôpitaux, écoles spécialisées et camps de vacances voués à la réadaptation et à l'éducation des " petits infirmes ", lesquels sont le plus souvent issus de la classe ouvrière.

La tragédie de la Thalidomide de 1961 accélère l'investissement symbolique, financier et institutionnel des gouvernements envers l'enfance handicapée. Cette deuxième phase est marquée par une logique de réparation, puisque l'État avait autorisé l'utilisation de la Thalidomide. Cette tragédie n'entraîne toutefois pas nécessairement une transformation profonde des attitudes sociales face à l'enfance handicapée et permet même de faire ressortir certaines continuités ayant principalement trait au rôle des mères d'enfants handicapés. Cette période est marquée par une certaine expansion des services offerts à une enfance dont les handicaps sont définis de manière de plus en plus diversifiée. La question des victimes de la Thalidomide et des ressources déployées pour les soutenir aide à explorer la période et illustre le changement d'optique qui marque les investissements étatiques et scientifiques accrus durant cette période.

Un cas particulier ressort toutefois dans l'examen de cette deuxième phase, même si sa présence marque l'ensemble de la période couverte par le volume, soit l'éducation des enfants épileptiques, trop souvent exclus des classes ordinaires à cause des réactions que provoquent autour d'eux les crises dont ils sont victimes. Il ne s'agit pas à proprement parler de handicap physique et là se pose une question que l'auteure évoque sans vraiment y répondre : qu'en est-il des enfants dont le handicap est autre qu'orthopédique? Chercher à explorer l'expérience des enfants marqués par la différence physique et mentale représente un défi considérable qu'il aurait été impossible de couvrir en une seule monographie, mais on peut se poser la question du choix de " handicaps " présentés dans ce volume, puisque les attitudes sociales dont l'analyse forme la trame de fond de ce volume s'appliqueraient aussi aux personnes sourdes, aveugles (que l'auteure a déjà étudiées dans un autre volume) et frappées par d'autres « déficiences " perçues.

Ce constat brouille aussi un peu les cartes quant à la transition entre cette phase de " réparation " et celle qui suit et que l'auteure dit opérer selon une logique «sociétale ». La grande différence ici relève d'un activisme croissant au sein des groupes de personnes handicapées elles-mêmes, ainsi que de groupes de parents, qui militent en fait pour que les enfants handicapés soient considérés comme des personnes à part entière et non encarcanés par des diagnostics définis par des médecins et éducateurs. Cette période, qui s'amorce de manière de plus en plus visible dans les années 1970 et qui prend de l'ampleur après l'Année internationale pour les personnes handicapées en 1981, amène une réorientation des modèles d'intervention axés sur l'intégration plutôt que sur l'exclusion. L'auteure explore ici la disparition ou la conversion des institutions spécialisées et l'apparition de modèles d'intervention individualisés, entraînant une expansion des services offerts, dans un cadre de resserrement budgétaire. Malgré l'intervention de groupes de pression et la transformation des institutions, l'auteure constate que les préjugés face au handicap ont la vie dure et qu'il ne suffit pas de changer les modèles d'intervention pour que les droits des enfants en situation de handicap soient respectés. Cela l'amène à conclure sur l'impact de crises sociales comme la Covid-19 sur les personnes handicapées. 
Cette étude se nourrit d'un abondant corpus documentaire auquel s'ajoutent les témoignages de onze témoins de diverses époques couvertes par cette étude. Ces récits émaillent l'analyse et lui donnent une dimension humaine rarement vue dans les études tirées exclusivement d'archives institutionnelles. Il s'agit là de l'un des nombreux éléments qui donnent à ce volume une valeur qui dépasse sa simple contribution à l'élargissement du savoir sur un secteur méconnu de l'histoire et qui en font un modèle à suivre. L'inclusion explicite de facteurs intersectionnels comme l'influence du genre et de la classe sociale dans l'analyse la rend également d'autant plus utile pour la recherche future.

Stéphane-D. Perreault 